\title{
Envejecimiento, bases para una titulación
}

\author{
Aging, bases for a degree
}

Envelhecimiento, bases paraum diploma

Miriam Costabel García ${ }^{1}$, Ma. Isabel Silva Quiroga ${ }^{2}$

\section{Resumen:}

OPS en su Serie Recursos Humanos para la Salud No 59informa las dificultades para formar RRHH en Gerontología Geriatría (G-G) dados por aspectos educativos, escasas ofertas laborales y centros asistenciales específicos. El saber en el tema es vasto, no es así el conocimiento que se tiene frente a la problemática en la formación de RRHH. identificada en la serie No 59. El objetivo como formadores de enfermeros posgraduados que dejo en "Stand by" una especialidad en G-G, estructurada en la diversidad de la problemática del adulto mayor AM cuyo curriculum oculto no colmó las expectativas de la comunidad educativa. La inquietud como profesores, gestores educativos de enfermería, en un departamento que profundiza los saberes sobre le cuidado AM, está en buscar que el programa perfile a un profesional con valores, competencias para el cuidado, contextualizado al Sistema Nacional Integrado Salud (SNIS), lenguaje analítico-crítico y virtual, transdiciplinar entre otras. Que contribuyan a la calidad de vida del AM, la familia, los aspectos sociales, el hábitat. Además de conocer la opinión de los licenciados clínicos. La revisión descriptiva de investigaciones de estudiantes y docentes del departamento en el período 2015-2019 dan cuenta de enunciados textuales y conceptos útiles para este fin, que conjugado a la experiencia docente informa sobre la tendencia buscada. ¿Qué información se ha recogido en las Tesis Finales de Investigación (TFI) que puedan enriquecer una nueva propuesta de formación posbásica en G-G.?

La demografía, la experticia de los profesores, la estrategia de posgrados articulados fundamenta la necesidad de un programa posbásico adaptado, que contemple al "life long learning", un aspirante en G-G que aprende métodos para aprender durante toda su vida.

${ }^{1}$ Magister Educación. Ex Prof. Facultad de Enfermería Udelar. Miembro Comisión Ad Hoc de Acreditación MEC. ORCID: 0000-0001-9013-8916.

e-mail: miriam.costabel@gmail.com

${ }^{2}$ Prof. Facultad de Enfermería. Udelar. Magister en Gestión Servicios de Salud. Director de Departamento Salud del Adulo y Anciano. ORCID: 0000-0002-9849-4920.

e-mail:silvaqisabel@gmail.com 
La proyección para el 2050 en el Uruguay es de casi 825.000 AM en una población de 3,7 millones, mientras que los menores de 15 serán más de 570.000 la epidemiologia misma demandará cuidado enfermero y cambios en la seguridad social.

\section{Palabras clave:}

Geriatría, Enfermería, Educación de Postgrado en Enfermería, Uruguay.

\section{Abstrac:}

PAHO in its Human Resources for Health Series No. 59 reports the difficulties to train HR in educational aspects, few job soffer and specific care centers in Gerontology Geriatrics (GG) Knowledge on the subject is vast, not so the knowledge that one has in front of the problem in the formation of RR. identified in series No 59. The objective as postgraduate nurse educators who left a specialty in G-G in "Stand by", structured in the diversity of the problems of the elderly AM whose hidden curriculum did not exceed the expectations of the educational community. The concern as teachers, nursing educational managers, in a department that deepens knowledge about AM care, is to seek that the program profiles a professional with values, skills for care, contextualized to the SNIS, analytical-critical and virtual language, transdisciplinar y among others. That they contribute to the quality of life of the MA, the family, the social aspects, the habit. In addition to knowing the opinion of clinical graduates.

The descriptive review of investigations of students and teachers of the department in the period 2015-2019 reveal textual statements and useful concepts for this purpose, which together with the teaching experience reports on the trend sought. What information has been collected in the TFIs that can enrich a new proposal for post-basic training in G-G?

The demographics, the expertise of the professors, the articulated postgraduate strategy support the need for an adapted post-basic program, which includes the "lifelong learning", a G-G candidate who learns methods to learn throughout his life.

The projection of Uruguay (almost 825,000 AM) in a population of 3.7 million, while those under 15 will be more than 570,000 the epidemiology itself will demand nursing care and changes in social security.

\section{Keywords:}

Geriatrics, Nursing, Graduate Education in Nursing, Uruguay. 


\section{Resumo:}

A OPAS, em sua Série de Recursos Humanos em Saúde $n^{\circ} 59$, relata as dificuldades para formar RH em aspectos educacionais, poucas ofertas de empregos e centros de atendimento específicos em Gerontologia Geriatria (GG) O conhecimento sobre o assunto é vasto, não o que se tem. diante do problema na formação do RR. identificados na série $n^{\circ} 59$. O objetivo como pós-graduandos enfermeiros educadores que saíram de uma especialidade em G-G em "Stand by", estruturados na diversidade dos problemas do idoso AM cujo currículo oculto não superava as expectativas da comunidade educacional. A preocupação como docentes, gestores do ensino de enfermagem, em um setor que aprofunda conhecimentos sobre o cuidado em AM, é buscar que o programa perfile um profissional com valores, habilidades para o cuidado, contextualizado ao SNIS, linguagem analítico-crítica e virtual , transdisciplinar entre outros. Que contribuam para a qualidade de vida do MA, da família, dos aspectos sociais, do hábito. Além de saber a opinião dos graduados em clínica. A revisão descritiva de investigações de alunos e docentes do departamento no período 20152019 revelam enunciados textuais e conceitos úteis para esse fim, que juntamente com a experiência docente relata a tendência buscada. Quais informações foram coletadas no TFI que podem enriquecer uma nova proposta de treinamento pós-básico em G-G?

A demografia, a expertise dos professores, a estratégia articulada da pós-graduação sustentam a necessidade de um programa pós-básico adaptado, que inclui o "life longlearning", um candidato do G-G que aprende métodos para aprender ao longo da vida.

A projeção de no Uruguai (quase 825.000 AM) em uma população de 3,7 milhões, enquanto os menores de 15 anos serão mais de 570.000 a própria epidemiologia exigirá cuidados de enfermagem e mudanças na previdência social.

\section{Palavras-chave:}

Geriatria, Enfermagem, Educação de Pós-Graduação em Enfermagem, Uruguai.

\section{Introducción}

En el presente ensayo se hace una interpretación a una línea de interés sobre la formación posgraduada del Licenciado en Enfermería sobre gerontología y geriatría. La perspectiva teórica se sustenta en la demografía, la epidemiologia de las personas mayores, las nuevas tendencias de aprendizaje de las nuevas generaciones, las demandas de la ciencia tecnolo- gía y salud, políticas sociales contributivas a la implementación de los planes de estudios, el concepto de transdisciplina desafían la elaboración de nuevas curriculas para la posgraduación. Se conjugan algunos datos resultados de indagaciones una línea de investigación de Departamento de Salud de Adulto y Anciano de la Facultad de Enfermería de la Universidad de la 
República. Además de contemplar lineamientos como el Plan Internacional de Acción sobre el Envejecimiento, surgido de la Segunda Asamblea Mundial (Madrid, 2002) observa: "Existe en todo el mundo una necesidad imperiosa de ampliar las oportunidades educacionales en la disciplinagerontología y geriatría para los profesionales de la salud".

\section{Desarrollo}

La Red de Enfermería Salud del Adulto Mayor (OPS), sugirió en su Plan de trabajo que los países miembros, conocieran el estado de situación de las necesidades de formación en su contexto. OPA en su Serie Recursos Humanos para la Salud No 59 informa las dificultades para formar RRHH desde el sistema educativo y de los mismos profesionales que no encuentran en el tema interés. El saber existente del tema es vasto, no es así el conocimiento que se tiene frente a la problemática en la formación de recursos humanos identificada en la serie No 59. La ocupación como formadores de profesionales enfermeros posgraduados se centró en proponer una especialidad en Gerontología y Geriatría, estructurada en la diversidad de la problemática del adulto mayor en un plan modulary fue dejado en "Stand by" procurando identificar mediante una serie de investigaciones la tendencia para una nueva titulación.

La inquietud nuestra como gestores educativos de una facultad esta en buscar que el programa perfile a un profesional con valores, conductas y ética, manejo de la comunicación, competencias para el cuidado, clínicas actualizadas en su metodología, prácticas inherentes al contexto del SNIS, habilidades comunicacionales en el lenguaje analítico-crítico y lenguaje virtual, entre otras habilidades. Con un curriculum adaptado al contexto y conocer el estado de situación y opinión de los propios licenciados.

La revisión descriptiva de los resultados de una línea de investigación de estudiantes y docentes del departamento en el período 2015-2019 creada a ese fin, dan cuenta de enunciados textuales y conceptos útiles para la enseñanza y el conocimiento del tema, que conjugado a la experiencia docente nos informa de la tendencia buscada. ¿Qué información se ha recogido en las TFI que puedan enriquecer una nueva propuesta de formación posbásica en G-G.? Se parte conceptualmente de que la curricula debe ser flexible, que sostenga una articulación de las políticas y los programas de salud, no obstante, el sustento teórico no contribuyó en el pasado a la elaboración de un curriculum de especialidad que respondiera al contexto, interesara a docentes, ni a los propios licenciados aun con un eje basado en el cuidado y una epidemiologia integral.

La tendencia teórica en la elaboración de los planes de estudio está centrada en competencias, ha sido demostrada por innumerables estudios, ya que estos se adaptan a la forma de aprender a los que van dirigidos. Estudios tales como las 10 tendencias en educación con competencias del Siglo XXI desarrollado por el Instituto de Economía Digital (ICEMD) de ESIC Business \& Marketing School analizado por el Comunicador Social Andrés Moncada describe las habilidades y capacidades de aprendizaje de los nuevos estudiantes.

Si se entiende que una competencia es una capacidad aprendida donde una persona puede resolver con creatividad, reflexivamente como lo cita Gómez(2017) una duda, una pregunta crítica, una actividad, un problema dando res- 
puesta a una situación real y asociar el concepto a la destreza con que los jóvenes operan los dispositivos tecnológicos. Las búsquedas con ingenio "powerpupils" o alumnos empoderados o aceptar que aceptar que "los estudiantes ya no son lo que eran son sujetos operantes, no objetos pasivos", los escenarios universitarios, son desafiados a cambiar los métodos de enseñanza y no solo la estructura de los curriculum pues el estudiante es un "life long learning" y aprende métodos para aprender durante toda su vida.

El interés en este proceso de conocer elementos contribuyentes para la elaboración asertiva de un nuevo Plan de posgraduación en G-Gy está sustentado en varias premisas. Es así que pueden identificarse algunas como la propia epistemología, es decir el deber ser del saber enfermero sobre el cuidado que impera en el Departamento sobre la persona mayor; en nuevas estrategias pedagógicas, en la situación demográfica del país, en el deber con la sociedad sobre su cuidado y formación que luce en la misión de la institución. La Ley de de ejercicio profesional de Enfermería $\mathrm{N}^{\circ} 18.815$; en su reglamentación expresa además en el artículo II, “.. con la finalidad de asegurar la calidad de los cuidados dirigidos a las personas, familias y comunidades, y la mejora continua como un eje del Sistema Nacional Integrado de Salud"; para asegurar la calidad entre otras cosas hay que formar.

Uruguay presenta una población envejecida, en un análisis prospectivo, proyectado al año 2050 la Oficina de Planeamiento y Presupuesto (OPP), plantea un escenario posible al 2050 con las tendencias actuales sin grandes cambios, llegando a una esperanza de vida de 82 años, con crecimiento significativo de la población de 65 años y más, que llega al 22,2\% del total (casi
825.000 adultos mayores) en una población de 3,7 millones, mientras que los menores de 15 serán algo más de 570.000 .

El Sistema Nacional Integrado de Salud (SNIS, 2008), ofrece cobertura médica a través de FONASA que incluye a pasivos mayores de 65 años y controla la equidad en prestadores de salud. El Programa Nacional Adulto Mayor (PRONAM) promueve la calidad de vida articulando acciones entre Estado (BPS, MIDES) y sociedad.

A través del análisis conceptual como herramienta metodológica (Scriven, 1988; Rico, 1999), se trabajó con enunciados textuales de los resultados de cada uno de los problemas de investigación definidos por cada TFI. Cada dato aportado fue cotejado con las dificultades identificadas en la Serie 59 pp. 11.Los trabajos revisados usaron como descriptores términos tales como anciano, envejecimiento, enfermería geriátrica, competencia, enfermería.

Altez, Y., (2020) se puede inferir de los marcos teóricos que las políticas en el Uruguay son protectoras de las Personas Mayores, no obstante, en el país hay un Hospital Centro Geriátrico "Hospital Centro Geriátrico "Dr. Luis Piñeyro del Campo" específico dependiendo de la Administración de Servicios del Estado el resto de los Hospitales del SNIS son polivalentes. Aspecto que dificultaría los escenarios de práctica para la posible implementación de la titulación, problema coincidente con lo identificado en por OPS en Latinoamérica. No obstante, existen locaciones para Adultos Mayores en Instituciones como hogares, residencias, otras reguladas por ley la cual no exige una gestión con Enfermeras tituladas. Los perfiles ocupacionales de las Enfermeras según el Censo Enfermería 2013 no muestra especialización en gerontología, ni ge- 
riatría, las ofertas de formación son escasas, las hubieron, pero no fueron sostenidas en el tiempo, los propios servicios de salud que asisten a la población no ofrecen capacitación sostenida y obligatoria. Andreatta, C. et al (2020) en su investigación de corte descriptivo los cuales encuestaron a 82 licenciados de tres diferentes zonas geográficas del país, solo el $30 \%$ hizo alguna formación de pocas horas en geriatría, $63 \%$ lo hicieron en la institución que trabajan y consideran el 98\% que los estudios de grado los han capacitado para la atención de la persona mayor, en $82 \%$ aplica por ejemplo la Valoración Integral Geriátrica (VGI). El Censo Enfermería 2013 mostró una escases en la relación de licenciados enfermeros por cama, los profesionales son 3.946 para 3.285.877 habitantes “...deja al descubierto y profundiza un déficit cuantitativo de profesionales y algunos desbalances en la composición de la fuerza de trabajo de la Enfermería del país." Pérez. M. (2015).

Sanguinet D. et al (2020)en otra investigación descriptiva encuestaron a 26 enfermeras profesionales muestreo a conveniencia, del área pública y privada y el $68 \%$ de ellas expresan no tener interés de dedicarse a la gerontología, se consideran capacitadas 81\%, no obstante, el 54\% no aplica la Valoración Geriátrica Integral, marcando una condición poco asertiva. En el medio son escasos los protocolos universales para atender a la PM otro aspecto coincidente con el identificado en la Serie 59.

Si se analiza el Censo Enfermero 2013, las especializaciones en cuidados críticos, emergencia y gestión son las que presentan mayor porcentaje, de lo cual puede inferirse el interés de los profesionales por estas especializaciones y la demanda, oferta del mercado laboral. La Serie 59 se expone como problema el doble empleo para que los licenciados opten por dedicarse a cuidado del PM en Latinoamérica, no obstante, en Uruguay respecto al trabajo solo el $34 \%$ tiene doble empleo en el nivel licenciados lo que diferencia la premisa de OPS.

El programa de formación posbásica debería estar centrado en una Maestría de Práctica Avanzada (EPA) entendiendo por ello como “... una enfermera / que ha adquirido una base de conocimientos especializados, habilidades complejas de toma de decisiones y habilidades clínicas para la práctica ampliada, cuyas características se moldean por el contexto y / o país en el que está acreditado para la práctica. Se recomienda el título de maestría para el nivel de entrada”. Según Dra. Silvia Cassiani las iniciativas de la OPS /OMS apuntan a apoyar ampliamente la implementación de (EPA), facilitando la colaboración y cooperación técnica con países y organizaciones nacionales de enfermería.

En cuanto a contenidos el plan debería estar estructurado en competencias como se expresó anteriormente, pero si se interpreta el estudio de revisión bibliográfica integral de Altez, Y. et al (2020) los cuales revisaron 9095 artículos donde 11 describían con mayor frecuencia competencias tales como comunicación, gestión, ética, conocimientos propios que fundamentan los cuidados enfermeros y habilidades técnicas. Estos conceptos textuales son los hilos conductores del Departamento en su proceso de enseñanza aprendizaje, los cuales pueden resumirse en -saber que- se tienen definido los contenidos a enseñar en el grado y que se pro- 
fundizarían y complementarían en el posgrado, -saber cómo- que son las técnicas y tecnologías, habilidades de pensamiento crítico, practicas reflexivas, uso de la tecnología y por ultimo -saber porque- que es la ética como eje y el respeto con que ejerce la profesión.

Se puede inferir que estas habilidades, definiciones de Mauk K. imperan, estas describen una serie de competencias susceptibles de ser integradas en el plan de pos graduación en G-G, tienen el aval de la Asociación estadounidense de Facultades de Enfermería.

En cuanto a la implementación se entiende que el programa debe procurar la coordinación interfacultades, fuerzas vivas, organismos gubernamentales y no gubernamentales que llevan adelante en forma conjunta la elaboración, ejecución y evaluación de programas de salud del adulto mayor, que a su vez sirvan de marco referencial para la enseñanza contextualizada de la enfermería en la titulación posbásica en G-Glo cual garantizaría la calidad de la propuesta.

¿Surge luego de ensayar las premisas la cuestión si se podrá mejorar el modelo asistencial enfermero-relacional para que sea el campo de la disciplina atractivo? ¿Qué relación tiene el concepto que poseen los licenciados sobre el envejecimiento que incidan en la no elección de este campo laboral, será que los puestos de trabajos no son redituables o no hay oferta, las causantes del desinterés?

Innumerables son los cuestionamientos aun por investigar. Los autores entienden que la necesidad de la formación, es casi una obligación y prosperarán en esta propuesta, que se tienen las capacidades y experticia en el tema, que se ha identificado la estrategia de redacción de la curricula, que se pueden salvar algunas dificul- tades descriptas por OPS y que el SNIS pueda empoderar la nueva titulación a la necesidad del cambio sustantivo de la demografía de los usuarios. La demografía, la experticia de los profesores del departamento de Salud del Adulto y Anciano, la estrategia de posgrados articulados Inter facultades que ofrece la Universidad de la República, la responsabilidad social que tiene la Institución declarada en su misión, la necesidad en salud de la población que se sostiene un envejecimiento de un $18.7 \%$ (INE), las nuevas tendencias educativas de formación por competencias, las nuevas tecnologías de la educación virtual y simulada, la demanda de cuidado calificado enfermería frente a las demandas epidemiológicas fundamentan la necesidad de un programa posbásico adaptado, que contemple al "life long learning", un aspirante de posgrado en GG que aprende métodos para aprender durante toda su vida.

Porque no hay cargos específicos para posgrados con formación en G-G o estructuras asistenciales específicas numerosas que el salario no sea atractivo no es motivo para negar una realidad que impera, existirán (casi 825.000 adultos mayores) en una población de 3,7 millones, mientras que los menores de 15 serán algo más de 570.000 la epidemiologia misma demandará cuidado enfermero y cambios en la seguridad social. 


\section{Referencias Bibliográficas}

1. Organización Panamericana de la Salud. Enseñanza de la enfermería en salud del adulto mayor. [Internet]. 2012. Disponible en: https://www.paho.org/hq/dmdocuments/2012/nursing-salud-adulto-mayor2012-spa.pdf [consulta: 14 nov 2020].

2. Declaración Política y Plan de Acción Internacional de Madrid sobre el Envejecimiento. [Internet]. 2002. Disponible en: https://social.un.org/ageing-working-group/ documents/mipaa-sp.pdf [consulta: 15 oct. 2020].

3. Reglamentación de la Ley $\mathrm{N}^{\circ} 18.815$ relativa a la regulación del ejercicio de la profesión universitaria de enfermería y de auxiliar de enfermería. Decreto No 354/014 de 4 de diciembre de 2014. Considerando II. [Internet]. Uruguay. Disponible en:https://www. impo.com.uy/bases/decretos/354-2014 [consulta: 14 nov 2020].

4. Uruguay. Presidencia de la República. Oficina de Planeamiento y Presupuesto. Hacia una estrategia nacional de desarrollo, Uruguay 2050. [Internet]. 2018. Disponible en: https://www.opp.gub.uy/sites/default/ files/documentos/2018-05/2257_Escenarios_demograficos_Uruguay_2050-_web. pdf [consulta: 10 oct. 2020].

5. Ramos G, Chiva I, Gómez MB. Las competencias básicas en la nueva generación de estudiantes universitarios: una experiencia de innovación. Revista de Docencia Universitaria [Internet] 2017; 15(1): 37-55.Disponible en: https://polipapers.upv.es/index.php/REDU/article/view/5909/7947 [consulta: 14 nov 2020].
6. Rico L. Análisis conceptual e investigación en didáctica de la matemática. [Internet]. 2001. Disponible en:https://www.uv.es/ aprengeom/archivos2/homenaje/12RicoL. PDF [consulta: 13 nov 2020].

7. Mauk KL.Enfermeríageriátrica: competencias asistenciales. [Internet]. 2008. Disponible en:http://docshare01.docshare.tips/ files/27044/270440913.pdf [consulta: 10 nov 2020].

8. Enfermería en el Uruguay 2015.OPS Censo Enfermeria.2015. Disponible en: https:// www.paho.org/es/documentos/enfermeria-uruguay-2015 [consulta: 14 nov 2020].

9. Altez Y, Martínez G, Martirena G, Páez MJ, Rodríguez A. Competencias específicas del Licenciado en Enfermería en la atención de los adultos mayores. Montevideo:UdelaR, FE;2020.

10. Andreatta C, Baptista M, Jamen S, Moar M, Pereyra L.Descripción de la situación de las competencias del Licenciado en Enfermería en el cuidado de las personas mayores hospitalizadas. Rivera: UdelaR, FE; 2020.

11. Sanguinet D, Moreira M, Pimentel N, Ehmer S. Competencias de las Licenciadas en Enfermería Gerontológica en los Centros Asistenciales de la Ciudad de Rivera. Rivera: UdelaR, FE; 2020.

Recibido: $21 / 11 / 20$

Aceptado: 12/04/21 